\title{
Myocardial cell therapy at the crossroads
}

\author{
Bernardo Nadal-Ginard and Valentin Fuster
}

In 2001, the first reports documenting postinfarct myocardial regeneration in rodents using bone-marrow-derived putative stem cells (BMDSCs) elicited great scientific and clinical interest. Despite the interest generated, however, these early reports neither identified the putative therapeutic cells nor provided data on dose-effect relationships or optimal administration parameters. Furthermore, they provided no insight into the mechanism or mechanisms responsible for the differentiation of BMDSCs into myocardial cells. An arduous program of animal experimentation was envisaged before the first clinical trials.

Surprisingly, within 6 months of the rodent study's publication, a phase I, nonrandomized clinical trial of BMDSC myocardial transplantation was published that reported a modest improvement in ejection fraction (EF). Unfortunately many investigators interpreted this publication as the starting shot for a stampede of clinical trials transplanting or mobilizing BMDSCs into human myocardium-without prior validation in animal models.

Over a dozen phase I trial findings of BMDSC-based therapy are now in the public domain-including three large randomized studies recently published in the New England Journal of Medicine. The results reported so far are modest at best, inconsistent and unconvincing. Moreover, although most study groups report an early, modest improvement in EF with BMDSC-based therapy, the difference in the EF change among the different trial's control groups is at least as large as that among control and active-treatment groups from the same trial. Even if the reported improvements are real, their clinical significance is highly doubtful.

This messy situation has been caused, at least partly, by the lack of meaningful preclinical data. Although most published trials with BMDSCs were designed to produce myocardial regeneration, the paucity of results has spawned a new interpretation: that BMDSCs have a 'paracrine

\section{We cannot \\ evaluate the \\ results of a \\ therapy when \\ we haven't \\ identified the \\ therapeutic \\ agent, its \\ mechanism \\ of action or \\ the most- \\ efficacious \\ doses.}

B Nadal-Ginard is a senior investigator at Mount Sinai School of Medicine, New York, NY, USA and Head of Research at the Centro Nacional de Investigaciones Cardiovasculares in Madrid, Spain, and $V$ Fuster is the Editorin-Chief of Nature

Clinical Practice

Cardiovascular Medicine.

\section{Competing interests}

The authors declared they have no competing interests.

www.nature.com/clinicalpractice doi:10.1038/ncpcardio0743 effect' on the surviving myocardium and also stimulate 'neovascularization' of the scar. Amazingly, this interpretation is invoked in peer-reviewed journals without a scintilla of evidence.

Another major weakness of the clinical trials published so far is that, on the basis of the best available evidence, no report has documented the myocardial regenerative capacity of either the cell mixtures or the constituent cells that have been transplanted into human myocardium. Moreover, assuming human and mouse cells to be equally efficient, the most optimistic calculations from rodent data indicate that the maximum amount of new myocardium generated during the clinical trials would be $1-5 \mathrm{~g}$. Since none of the methods for measuring ventricular function can measure the functional contribution of just $5 \mathrm{~g}$ of myocardium, however, it follows that no trial has transplanted sufficient BMDSCs to have a measurable effect.

We cannot evaluate the results of a therapy when we haven't identified the therapeutic agent, its mechanism of action or the mostefficacious doses. We, therefore, suggest a moratorium on new clinical trials until information needed to properly evaluate the results has been obtained and important questions have been answered: which cells produce the putative beneficial effect? What is the optimal method of administration of the cells to produce an effect on human-sized hearts? In what mechanism or mechanisms of action are the transplanted cells involved?

On the basis of the available experimental evidence and the new understanding of the role of cardiac stem cells as regulators of myocardial cell homeostasis, it is difficult not to be optimistic about the potential of myocardial regeneration research. Yet realizing this potential requires a broad consensus on the information needed before experimental animal data can be extrapolated to humans. 